\title{
Neutron star structure with a new force between quarks
}

\author{
Jeffrey M. Berryman $\odot^{*}$ \\ Department of Physics, University of California, Berkeley, California 94720, USA \\ and Department of Physics and Astronomy, University of Kentucky, Lexington, Kentucky 40506, USA \\ Susan Gardner $\oplus^{\dagger}$ \\ Department of Physics and Astronomy, University of Kentucky, Lexington, Kentucky 40506, USA
}

(Received 14 May 2021; revised 21 July 2021; accepted 1 September 2021; published 8 October 2021)

\begin{abstract}
The discovery of nondiffuse sources of gravitational waves through compact-object mergers opens new prospects for the study of physics beyond the Standard Model. In this paper, we study the effects of a new force between quarks, suggested by the gauging of baryon number, on pure neutron matter at supranuclear densities. This leads to a stiffening of the equation of state, allowing neutron stars to be both larger and heavier and possibly accommodating the light progenitor of GW190814 as a neutron star. The role of conventional three-body forces in neutron star structure is still poorly understood, though they can act in a similar way, implying that the mass and radius do not in themselves resolve whether new physics is coming into play. However, a crucial feature of the scenario we propose is that the regions of the new physics parameter space that induce observable changes to neutron star structure are testable at low-energy accelerator facilities. This testability distinguishes our scenario from other classes of new phenomena in dense matter.
\end{abstract}

DOI: 10.1103/PhysRevC.104.045802

\section{INTRODUCTION}

The environment of a proto-neutron star (NS) has long been known to be exquisitely sensitive to the appearance of new physics (NP), manifested through light particles such as axions [1-7] or dark photons [8-10], through cooling effects. Dark matter can also be captured by NSs, altering them so severely that the established existence of NSs constrains dark matter properties [11,12]. With the advent of gravitational wave (GW) observations of compact object mergers [13], new windows on the nature of matter at supranuclear densities open [14]. There has been much discussion of emergent phenomena within QCD at near-zero temperature $T$ with neutron chemical potential $\mu_{n}$, with transitions to condensed phases [15] of pions [16] and kaons [17], or to a spin-colorflavor-locked $q q$ phase $[18,19]$, or to states with substantial admixtures of $s$ and $\bar{s}$ quarks all possible [20-22]. The structure of NSs is also sensitive to new neutron decay channels, as noted in new physics models for the neutron lifetime anomaly [23,24], yielding severe constraints [25-27].

Here, we consider minimal extensions of the Standard Model (SM) that give rise to new, short-range interactions be-

\footnotetext{
*jeffberryman@berkeley.edu

†gardner@pa.uky.edu
}

Published by the American Physical Society under the terms of the Creative Commons Attribution 4.0 International license. Further distribution of this work must maintain attribution to the author(s) and the published article's title, journal citation, and DOI. Funded by SCOAP . tween quarks. In particular, we consider $\mathrm{U}(1)_{X}$ extensions that couple to baryon number $B$; such models have proven popular in searches for light hidden sectors in low-energy accelerator experiments [28] because the possibility that the neutrino has a Majorana mass predicates that the $B-L$ symmetry of the $\mathrm{SM}$ is broken. If $B$ symmetry is spontaneously broken to give a gauge boson $X$ no lighter than a few hundred $\mathrm{MeV}$, then the new interaction is largely shielded from constraints from low-energy experiments. In particular, its contribution to the nucleon-nucleon $(N N)$ force can be hidden within the short-distance repulsion of the phenomenological $N N$ force in the SM, recalling, e.g., the repulsive hard core of the Reid potential at separations of $r_{\mathrm{hc}}=0.5 \mathrm{fm}$ [29], yet it can modify the the neutron matter equation of state $(\mathrm{EoS})$ at supranuclear densities, i.e., beyond the saturation number density of ordinary nuclear matter, $n_{\text {sat }}$ [30]. We expect these models to be accompanied by electromagnetic signatures, such as, e.g., brighter kilonovas, due to $X-\gamma$ mixing, but reserve this for later work [31].

\section{THEORETICAL FRAMEWORK}

The lighter compact object in GW190814 is of $2.50-2.67 M_{\odot}(90 \%$ credible level) in mass [32], and is likely too heavy to be a NS, at least within a nonrelativistic many-body approach using $N N$ forces from chiral effective theory, with low-energy constants (LECs) determined from nuclear data [32]. Relativistic mean-field models can generate masses in excess of $2.6 M_{\odot}$ [33], though they are challenged by constraints from heavy-ion collisions (HICs) [34]; we note Refs. [35-56] for further discussion of the light progenitor 
of GW190814 as a neutron star. We consider our NP model within a nonrelativistic many-body framework. Drischler et al. [57] recognize the importance of relativistic corrections but also think that knowledge of the high-density EoS is likely inadequate. In particular, they adopt a piecewise EoS: beyond some density cutoff, the EoS is given by the stiffest form allowed by causality. They choose a cutoff density in the region $(1-2) n_{\text {sat }}$ and claim that the modified EoS and NS outcomes below that cutoff can be made without relativistic corrections.

The chiral effective theory approach uses $N N$ and nuclear data to determine the LECs, with independent two- and threebody forces coming into play [58,59]. In contrast, our Abelian NP model directly yields two-body forces only. To sharpen the distinction between SM and NP effects, we employ the AV18 $N N$ interaction [60], the properties of which are determined by $N N$ observables only. Studies of the AV18 interaction in pure neutron matter (PNM) with different nonrelativistic methods show that it compares favorably with other interactions up to about $4 n_{\text {sat }}$ [61]. Here, we compare computations of the PNM EoS using Brueckner-Hartree-Fock (BHF) theory with the AV18 interaction with and without NP.

\section{SECRET INTERACTIONS OF QUARKS}

That new interactions could exist between quarks is a longstanding possibility [62-66], and SM extensions in which a new vector mediator couples to baryon number $B[64,65,67-$ 70] yield a repulsive interaction between baryons. Thus we suppose a quark $q$ can interact via

$$
\mathcal{L} \supset \frac{1}{3} g_{X} \bar{q} \gamma_{\mu} q X^{\mu}
$$

Since quarks themselves carry electric charge, this generates $X-\gamma$ mixing at the quantum level. At $\mathrm{GeV}$-scale energies, we have

$$
\mathcal{L} \supset g_{B} \bar{N} \gamma_{\mu} N X^{\mu}+\varepsilon e \bar{N} \gamma_{\mu} \frac{\left(1+\tau_{3}\right)}{2} N X^{\mu}-\varepsilon e \bar{\ell}_{i} \gamma_{\mu} \ell_{i} X^{\mu}
$$

where $N$ is the nucleon doublet, $\ell_{i}$ is a charged lepton, and $\varepsilon$, although crudely $\approx e g_{B} /(4 \pi)^{2}$, can be made smaller still [71], with $\varepsilon \sim 10^{-8}-10^{-2}$ [72]. Experimental constraints are such that the new mediator cannot be too light $[64,70]$; we focus on gauge mediators of about $0.2-1 \mathrm{GeV}$ in mass, for which $g_{B}$ can be as large as $g_{B} \approx 0.4[65,70,73]$. However, if we adopt a $\mathrm{U}(1)_{B_{1}}$ model, so that the gauge boson couples to first-generation quarks only, constraints from hadronic $J / \psi$ and $\Upsilon$ decays are weakened as $X$ can only mediate these decays through kinetic mixing and $g_{B}$ can be $\mathcal{O}(1)$. Although $\varepsilon \neq 0$, dark photon searches limit $\varepsilon \lesssim 10^{-3}$ for $M_{X} \sim 1 \mathrm{GeV}$ [74]; we neglect all $\mathcal{O}(\varepsilon)$ effects in this paper. We emphasize that a $\mathrm{U}(1)_{B_{1}}$ model with the new interaction of Eq. (2) alone is incomplete: the addition of new fermions is required to make the theory consistent at high energies. Different completions are possible; we note Refs. [65,71,75-77] as examples. Other $\mathrm{U}(1)_{X}$ models [65,78] could give rise to Eq. (1) and thus be operative. For example, if $X=(B-L)_{1}$, then the theory is consistent if just one right-handed neutrino is added. The possibilities can be distinguished through direct or hidden sector searches at accelerator facilities, or through $v, e^{ \pm}$, or $\mu^{ \pm}$emission in compact object mergers.

\section{NEW NN FORCES IN NEUTRON AND NUCLEAR MATTER}

To evaluate the effect of the new $N N$ force in the nuclear medium and its modification to the EoS, we compute the effective two-particle interaction using the Brueckner-BetheGoldstone equation,

$$
G=V+\frac{V Q}{\omega-H_{0}} G,
$$

with $V$ the vacuum two-nucleon potential, $Q$ the Fermi operator, $\omega$ the initial two-particle energy, and $H_{0}$ the in-medium Hamiltonian. The $G$ matrix is then used to calculate the singleparticle potential $U(\mathbf{k})$ and the energy per nucleon $E / A$ of the system. Both $\omega$ and $H_{0}$ in Eq. (3) depend on the potential $U(\mathbf{k})$; one must ensure consistency between the potential used as input and that calculated from $G$, noting Refs. [31,79-81] for details.

We write the two-particle potential as $V=V_{\mathrm{AV} 18}+V_{\mathrm{NP}}$, where $V_{\mathrm{AV} 18}$ is that in Refs. $[60,82]$ and $V_{\mathrm{NP}}$ is generated by NP, being of Yukawa form:

$$
V_{\mathrm{NP}}=\frac{\alpha_{B_{1}}}{r} e^{-M_{X} r}
$$

with $\alpha_{B_{1}}=g_{B_{1}}^{2} / 4 \pi$ the strength of the gauged $\mathrm{U}(1)_{B_{1}}$ interaction and $M_{X}$ the mass of the associated boson. This interaction only ever stiffens the EoS. If the new boson is heavier than the pion and not too strongly coupled, then its effects on nuclear matter are expected to be subdominant to those of the strong interactions, comprising at most some part of the empirical LECs. For simplicity we approximate NSs as composed of PNM, noting that Table XI of Ref. [83] shows that the maximum NS masses in PNM and $\beta$-stable matter differ by less than $\approx 0.5 \%$. We evaluate Eq. (3) by decomposing it into its partial wave components and summing these contributions to the EoS. We consider partial waves up to $J_{\max }=11$, facilitating comparison with Ref. [61]. Considering only the AV18 potential, we obtain $E / A=13.7 \mathrm{MeV}$ at $n=n_{\text {sat }}=$ $0.16 \mathrm{fm}^{-3}$, compared to $13.4 \mathrm{MeV}$ [61]; for $n=0.3 \mathrm{fm}^{-3}$, we obtain $26.0 \mathrm{MeV}$, in perfect agreement.

A primary source of uncertainty in nuclear matter calculations is the precise many-body technique employed. For instance, Ref. [61] contrasts several such methods and finds that the in-medium potential energies may differ by a factor of $\approx 2$ at supranuclear densities for the same $N N$ potential. However, our purpose is to identify the allowed region of the $g_{B}-M_{X}$ parameter space in which the new interaction produces significant changes to NS structure. We assume that the answer to this question does not depend on the many-body method we use [84], and we emphasize that the uncertainties from three-body forces in this case are moot.

\section{RESULTS}

In Fig. 1(a), we show the NS mass-radius $(M-R)$ relationship of our BHF EoS; the dotted line considers only AV18, 

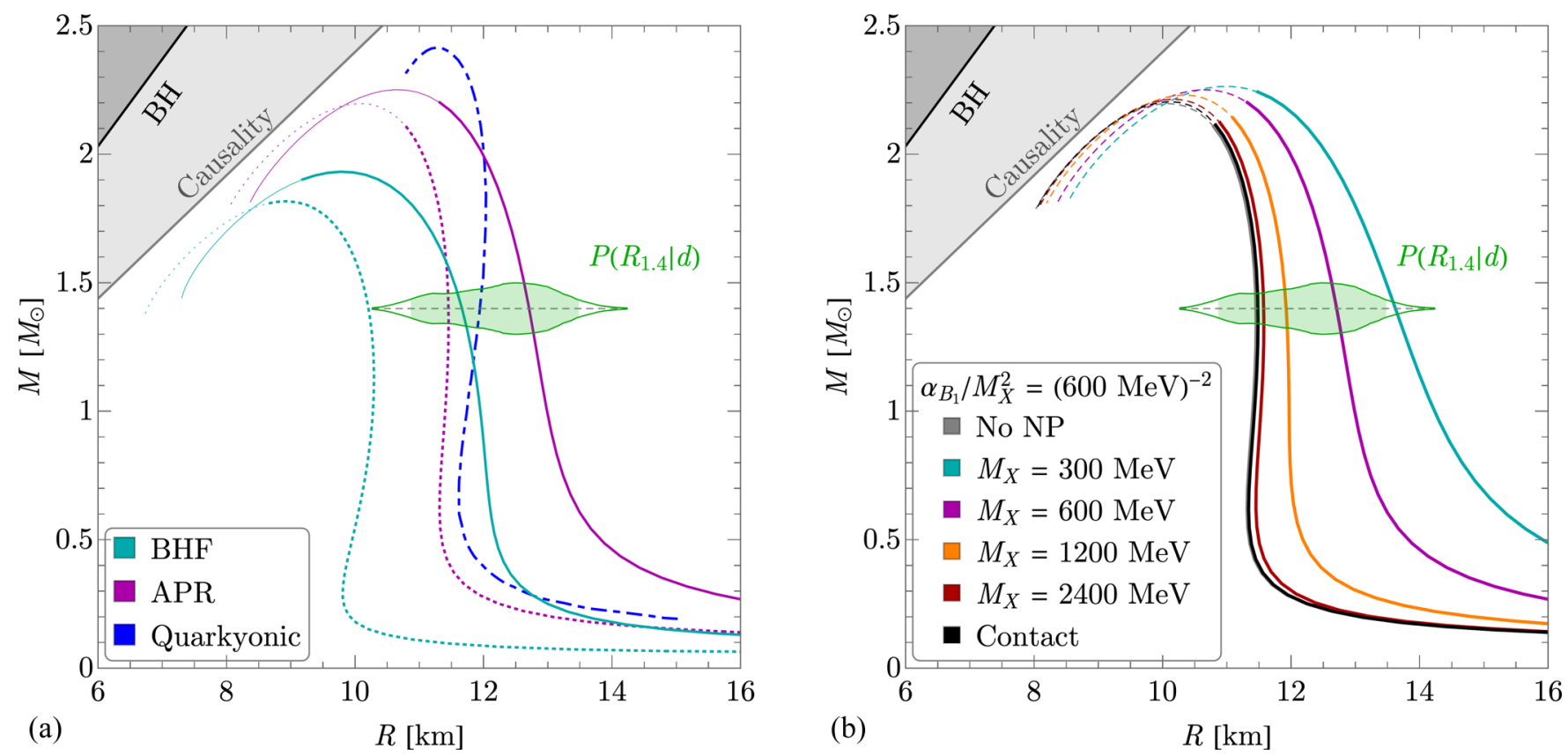

FIG. 1. (a) Mass-radius curves for our candidate EoS. Dotted lines assume only AV18 interactions [60]; solid lines introduce NP with $\alpha_{B_{1}}=1$ and $M_{X}=600 \mathrm{MeV}$. The dot-dashed line represents the quarkyonic EoS of Ref. [85]. (b) Mass-radius curves for fixed $\alpha_{B_{1}} / M_{X}^{2}=$ $(600 \mathrm{MeV})^{-2}$ for different values of $M_{X}$. In either panel, the violin region represents the PSRs+GWs+NICER posterior on $R_{1.4}$ after Fig. 10 of Ref. [86]; the shading indicates the $90 \%$ credible region.

whereas the solid line introduces NP with $\alpha_{B_{1}}=1$ and $M_{X}=$ $600 \mathrm{MeV}$. Thick lines indicate where the EoS is causal, i.e., the points for which the sound speed $c_{s}^{2} \leqslant 1$ in the core; thin lines indicate where it is not. The maximum stable, causal NS mass, $M_{\mathrm{TOV}}$, without NP is $\approx 1.8 M_{\odot}$ [87]; even with NP, this EoS is too soft to comfortably accommodate known heavy pulsar masses [88-91], 2.0-2.2M $\odot$. This is unsurprising, since this treatment neglects relativistic corrections and three-body forces. To remedy this, we have also considered the EoS presented in Ref. [83] ("APR"), which is built on the AV18 two-nucleon potential and the Urbana IX three-body potential [92]. We approximate NP effects on this EoS by calculating the difference between $E / A$ with NP and without it in the BHF scheme, and then adding this difference to the nominal APR EoS for PNM. The results are also shown in Fig. 1(a); the curves have the same interpretation as for the BHF case. Here we find $M_{\mathrm{TOV}} \sim 2.1 M_{\odot}$ - this is consistent with the mass of PSR J0740+6620, 2.14 ${ }_{-0.09}^{+0.10} M_{\odot}$ [91], but is still too light to explain GW190814. In both cases, the new interaction generates larger NS masses, as anticipated, but also increases the radius of NSs of a given mass.

To contextualize Fig. 1(a), we show the region in the $M-R$ plane in which the NS becomes a black hole, as well as a limit on NS properties from causality [93]. Moreover, we show the posterior probability on the radius of a $1.4 M_{\odot}$ NS, $P\left(R_{1.4} \mid d\right)$, conditioned on data $d$ from heavy pulsars, gravitational wave events, and NICER observations of PSR J0030+0451, adapted from Fig. 10 of Ref. [86]. Lastly, we have included the EoS derived for quarkyonic matter in Ref. [85] as an example with a quark/hadron QCD phase transition.The salient feature of this EoS is that the transition from hadronic to mixed hadron-quark matter induces a spike in $c_{s}^{2}$ at a few times saturation density; similar features are present in some of the sound speed profiles considered in Ref. [94]. This rapid stiffening of the EoS is sufficient to allow for heavier NSs and is consistent with inferences of $c_{s}^{2}$ [86] but is not strictly required. The new vector interaction induces a milder increase in $c_{s}^{2}$; the effect is that more severe increases in $M_{\mathrm{TOV}}$ are correlated with larger low-mass NSs. This prediction may be crucial for disentangling the presence of NP from critical QCD phenomena.

In Fig. 1(b), we fix the interaction strength to be $\alpha_{B_{1}} / M_{X}^{2}=$ $(600 \mathrm{MeV})^{-2}$ for four finite values of $M_{X}$. Also shown are the contact-interaction limit and the baseline APR EoS. Lighter states generate larger contributions to the EoS, and thus to NS properties, owing largely to their effects on higher partial-wave potentials. Contact interactions only contribute to $s$-wave scattering, whereas finite-mass states contribute at all orders. Because higher partial waves become more important at higher densities, the outcome is shown in Fig. 1(b). The pure contact interaction produces a result that is barely distinguishable from the nominal APR curve.

We also calculate the EoS of symmetric nuclear matter (SNM) using the same techniques with $J_{\max }=8$. In Fig. 2, we show the pressure determined in our BHF and APR schemes, and we include NP with $\alpha_{B_{1}}=1$ and $M_{X}=600 \mathrm{MeV}$. We compare these with inferences of the EoS from HIC, shown in shading. As with PNM, the pure BHF EoS is too soft to accommodate observations, but the APR EoS is a plausible candidate. The new interaction stiffens the EoS, as expected, but not so much that the HIC constraint is violated. For context, we show several mean-field EoSs [33,95-97]. We note that the pressure for $\mathrm{APR}+\mathrm{NP}$ is nonzero at empirical 


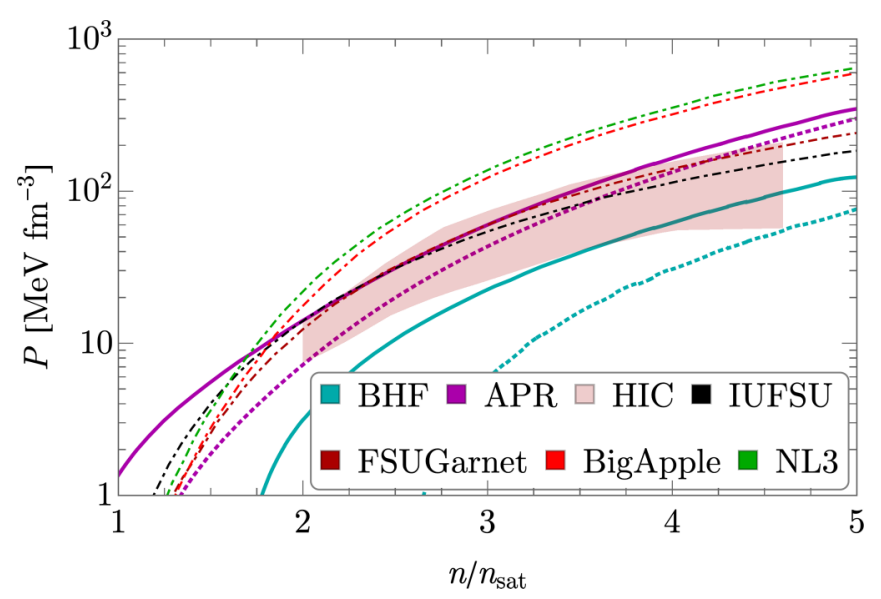

FIG. 2. A comparison between the pressures in our candidate EoSs for SNM and measurements from HIC data [34]. Dotted lines are for AV18 interactions; solid lines introduce NP with $\alpha_{B_{1}}=1$ and $M_{X}=600 \mathrm{MeV}$. We contrast these with mean-field calculations in Ref. [33] as dot-dashed lines.

saturation density; we expect this to be resolved with a more refined treatment, while still generating nontrivial effects at higher densities.

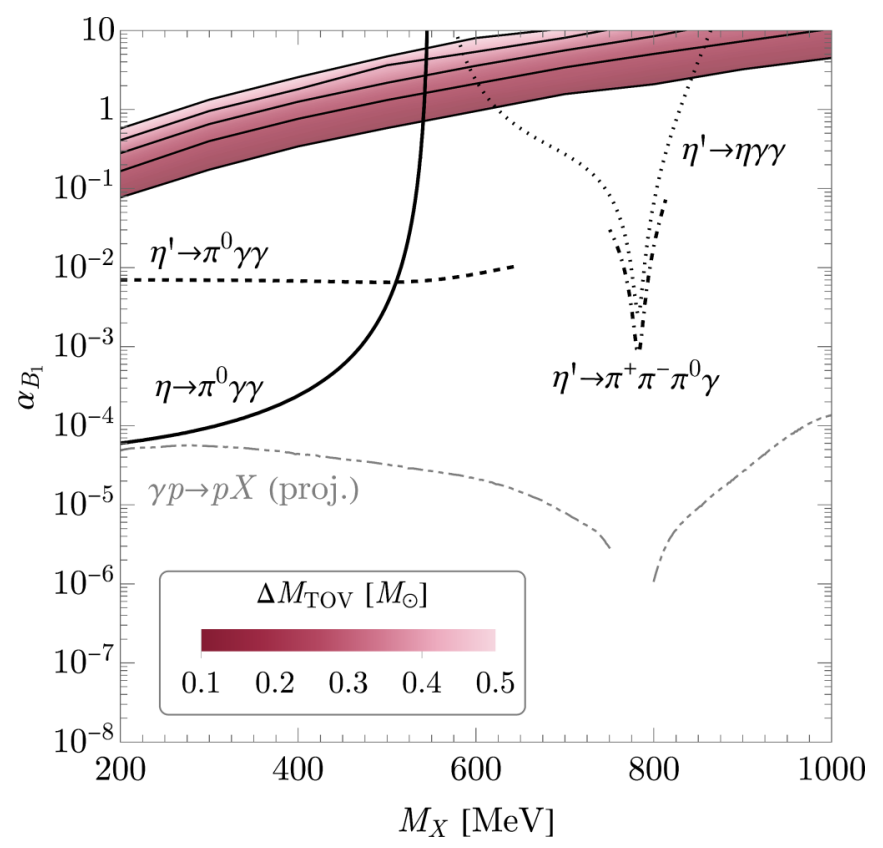

FIG. 3. Estimated constraints on the mass and coupling of a $U(1)_{B_{1}}$ gauge boson from $\eta^{(\prime)}$ decays assuming the SM contribution is zero, albeit disparate nonzero SM assessments exist [98-102]. Black lines are based on existing data [103] after Ref. [70] and the gray line is a projection as noted in text. Also shown is the change to the maximum TOV mass, $\Delta M_{\mathrm{TOV}} \in[0.1,0.5] M_{\odot}$; increments of $0.1 M_{\odot}$ are emphasized in black.

\section{CONSTRAINTS}

In Fig. 3, we show the region in the $\alpha_{B_{1}}-M_{X}$ plane in which $M_{\mathrm{TOV}}$ is increased by $0.1-0.5 M_{\odot}$ relative to the APR EoS. We now turn to potential constraints on this scenario from lowenergy physics. The presence of NP induces a contribution to the $N N$ scattering lengths. In the Born limit, the $n n{ }^{1} S_{0}$ scattering length is modified by

$$
\Delta a^{S_{S_{0}}}=\frac{\alpha_{B_{1}} m_{N}}{M_{X}^{2}} \approx(0.5 \mathrm{fm}) \times \alpha_{B_{1}}\left(\frac{600 \mathrm{MeV}}{M_{X}}\right)^{2} .
$$

We emphasize the AV18 potential is phenomenological-it is fit to low-energy $N N$ data, not derived from first principles. If the potential parameters were determined in the presence of NP, the effects of NP would presumably be obscured; we leave a detailed study to future work [31]. Therefore, low-energy $N N$ scattering does not provide a robust constraint on this scenario. New baryon-coupled physics can also be probed by lead-neutron scattering [104,105]. Reference [105] presents a constraint for masses below $40 \mathrm{MeV}$; Ref. [70] extends this into the $\mathcal{O}(100) \mathrm{MeV}$ mass range. However, if the range of the new force is not longer than the range of the nuclear force, then it is difficult to disentangle the two without a first-principles description of the latter, and the treatments of Refs. [104,105] are unsuitable to this mass region. As such, we do not consider this constraint further.

We calculate the contribution of our new boson to several rare $\eta^{(\prime)}$ decays using the vector meson dominance model [106-109]. We assume that the decays proceed via $\eta^{(\prime)} \rightarrow X \gamma, X \rightarrow \pi^{0} \gamma, \pi^{+} \pi^{-} \pi^{0}, \eta \gamma$, through $X-\omega$ meson mixing and that the SM contribution is zero. The observables are ratios of the rare decay widths to the widths for $\eta^{(\prime)} \rightarrow \gamma \gamma$ [103]. The solid curve in Fig. 3 shows the constraint derived from $\eta \rightarrow \pi^{0} \gamma \gamma$. Following Ref. [70], we require that the contribution from $X$ not exceed $3 \times 10^{-4}$ [103]; equality gives the curve shown. The SM contributions to $\eta^{(\prime)} \rightarrow \pi^{0} \gamma \gamma$ are not negligible; moreover, different width assessments exist [98-102] and further exploration is needed [31]. The upcoming JLab Eta Factory (JEF) experiment $[110,111]$ can perform a bump hunt in $\pi^{0} \gamma$ invariant mass, greatly enhancing the sensitivity to the $X$ gauge boson while mitigating sensitivity to the theoretical production rate [112].

Figure 3 also shows constraints from decays of $\eta^{\prime}$ to $\pi^{0} \gamma \gamma$ [113], $\pi^{+} \pi^{-} \pi^{0} \gamma$ [114], and $\eta \gamma \gamma$ [115]. The possibility of gluonium content in the $\eta^{\prime}[116,117]$ also complicates their interpretation. Analyses of neutral meson radiative decays do not agree on its size [118,119], where the inclusion of $\Gamma\left(\eta^{\prime} \rightarrow \gamma \gamma\right) / \Gamma\left(\pi^{0} \rightarrow \gamma \gamma\right)$ data drives this difference and a larger effect [119]. Our estimates assume this is zero, so that observed deviations between SM predictions and experiment could also derive from this effect. An alternative strategy for observing $X$ would be to search for bumps in invariant mass distributions in these decays. We caution, however, that there are regions in parameter space in which we expect the $X$ to be wide: this is so for $\alpha_{B_{1}} \gtrsim \mathcal{O}\left(10^{-1}\right)$ around the $\omega$ resonance, and for $\alpha_{B_{1}} \gtrsim \mathcal{O}(1)$ above $M_{X} \sim 1 \mathrm{GeV}$. In these regimes, the $X$ would not present as a localized feature in invariant mass and bump hunts would become less 
effective. These decays could be measured precisely at JEF and REDTOP [120-122], though the sensitivities have not been benchmarked. Additionally, the ultimate sensitivity of GlueX [123] to $X$ photoproduction $(\gamma p \rightarrow p X)$ affords a sensitivity to couplings of order $\mathcal{O}\left(10^{-5}-10^{-4}\right)$ for narrow $X$ off the $\omega$ resonance [124]; this is also shown in Fig. 3.

\section{SUMMARY}

We have considered how a new force between firstgeneration quarks can make NSs for a fixed EoS and many-body method both heavier and puffier. This mechanism has not been considered previously, though new forces for strange quarks have been considered [125]; the attractive $\Lambda \mathrm{N}$ interaction has long made the existence of $\approx 2 M_{\odot}$ NSs a puzzle [88], though three-body forces may reduce the effect [126]. We have described how our NP scenario can be tested through studies of rare $\eta$ and $\eta^{\prime}$ decays [112,122] and of $X$ photoproduction [124] at low-energy accelerators. Finally, although we have not resolved the nature of the $\approx 2.6 M_{\odot}$ compact object in GW190814, this mechanism allows it to more naturally be a NS. The spin of that object, though poorly determined, may have been sufficient to increase its mass by $\approx(0.1-0.4) M_{\odot}[35,36,52,127-132]$; differential rotation can push this even higher [133-135], but these configurations are not expected to be stable over long time scales. Combining spin effects with NP could yield additional heavy NSs; thus more compact objects in excess of $2 M_{\odot}$ may eventually be identified, promoting the possibility of new baryonic interactions.

\section{ACKNOWLEDGMENTS}

We thank the Network for Neutrinos, Nuclear Astrophysics, and Symmetries (N3AS) for an inspiring environment and support. J.M.B. acknowledges support from National Science Foundation Grant No. PHY-1630782 and Heising-Simons Foundation Grant No. 2017-228, and S.G. acknowledges partial support from U.S. Department of Energy Contract No. DE-FG02-96ER40989.
[1] N. Iwamoto, Axion Emission From Neutron Stars, Phys. Rev. Lett. 53, 1198 (1984).

[2] M. S. Turner, Axions from SN1987A, Phys. Rev. Lett. 60, 1797 (1988).

[3] R. P. Brinkmann and M. S. Turner, Numerical rates for nucleon-nucleon, axion bremsstrahlung, Phys. Rev. D 38, 2338 (1988).

[4] A. Burrows, M. S. Turner, and R. P. Brinkmann, Axions and SN 1987A, Phys. Rev. D 39, 1020 (1989).

[5] H.-T. Janka, W. Keil, G. Raffelt, and D. Seckel, Nucleon Spin Fluctuations and the Supernova Emission of Neutrinos and Axions, Phys. Rev. Lett. 76, 2621 (1996).

[6] C. Hanhart, D. R. Phillips, and S. Reddy, Neutrino and axion emissivities of neutron stars from nucleon-nucleon scattering data, Phys. Lett. B 499, 9 (2001).

[7] A. Sedrakian, Axion cooling of neutron stars, Phys. Rev. D 93, 065044 (2016)

[8] J. B. Dent, F. Ferrer, and L. M. Krauss, Constraints on light hidden sector gauge bosons from supernova cooling, arXiv:1201.2683 (2012).

[9] H. K. Dreiner, J.-F. Fortin, C. Hanhart, and L. Ubaldi, Supernova constraints on $\mathrm{MeV}$ dark sectors from $e^{+} e^{-}$annihilations, Phys. Rev. D 89, 105015 (2014).

[10] D. Kazanas, R. N. Mohapatra, S. Nussinov, V. L. Teplitz, and Y. Zhang, Supernova bounds on the dark photon using its electromagnetic decay, Nucl. Phys. B 890, 17 (2015).

[11] A. Gould, Resonant Enhancements in WIMP capture by the earth, Astrophys. J. 321, 571 (1987).

[12] S. D. McDermott, H.-B. Yu, and K. M. Zurek, Constraints on scalar asymmetric dark matter from black hole formation in neutron stars, Phys. Rev. D 85, 023519 (2012).

[13] B. P. Abbott et al., Observation of Gravitational Waves from a Binary Black Hole Merger, Phys. Rev. Lett. 116, 061102 (2016).

[14] F. J. Llanes-Estrada and E. Lope-Oter, Hadron matter in neutron stars in view of gravitational wave observations, Prog. Part. Nucl. Phys. 109, 103715 (2019).
[15] N. K. Glendenning, First order phase transitions with more than one conserved charge: Consequences for neutron stars, Phys. Rev. D 46, 1274 (1992).

[16] J. N. Bahcall and R. A. Wolf, Neutron stars. I. properties at absolute zero temperature, Phys. Rev. 140, B1445 (1965).

[17] D. B. Kaplan and A. E. Nelson, Strange goings on in dense nucleonic matter, Phys. Lett. B 175, 57 (1986).

[18] K. Rajagopal and F. Wilczek, The condensed matter physics of QCD, in At the Frontier of Particle Physics: Handbook of QCD, edited by M. Shifman (World Scientific, Singapore, 2001), pp. 2061-2151.

[19] M. Alford, Color-superconducting quark matter, Annu. Rev. Nucl. Part. Sci. 51, 131 (2001).

[20] N. K. Glendenning, Neutron stars are giant hypernuclei? Astrophys. J. 293, 470 (1985).

[21] C. Alcock, E. Farhi, and A. Olinto, Strange stars, Astrophys. J. 310, 261 (1986).

[22] F. Weber, Strange quark matter and compact stars, Prog. Part. Nucl. Phys. 54, 193 (2005).

[23] B. Fornal and B. Grinstein, Dark Matter Interpretation of the Neutron Decay Anomaly, Phys. Rev. Lett. 120, 191801 (2018).

[24] F. E. Wietfeldt and G. L. Greene, Colloquium: The neutron lifetime, Rev. Mod. Phys. 83, 1173 (2011).

[25] D. McKeen, A. E. Nelson, S. Reddy, and D. Zhou, Neutron Stars Exclude Light Dark Baryons, Phys. Rev. Lett. 121, 061802 (2018).

[26] T. F. Motta, P. A. M. Guichon, and A. W. Thomas, Implications of neutron star properties for the existence of light dark matter, J. Phys. G 45, 05LT01 (2018).

[27] G. Baym, D. H. Beck, P. Geltenbort, and J. Shelton, Testing Dark Decays of Baryons in Neutron Stars, Phys. Rev. Lett. 121, 061801 (2018).

[28] J. Alexander, M. Battaglieri, B. Echenard, R. Essig, M. Graham, E. Izaguirre, J. Jaros, G. Krnjaic, J. Mardon, D. Morrissey et al., Dark Sectors 2016 Workshop: Community Report, arXiv:1608.08632 (2016). 
[29] R. V. Reid, Jr., Local phenomenological nucleon-nucleon potentials, Ann. Phys. (NY) 50, 411 (1968).

[30] In effective field theory language, its effect is embedded within a low-energy constant at renormalization scales below $\mu<$ $M_{X}$, but $X$ becomes an active degree of freedom at scales above $\mu>M_{X}$. In the current context we suppose $X$ is explicit if $k_{F} c$ is not grossly smaller than $M_{X}$.

[31] J. M. Berryman and S. Gardner (unpublished).

[32] R. Abbott, T. D. Abbott, S. Abraham, F. Acernese, K. Ackley, C. Adams, R. X. Adhikari, V. B. Adya, C. Affeldt, M. Agathos et al. (LIGO Scientific, Virgo), GW190814: Gravitational waves from the coalescence of a 23 solar mass black hole with a 2.6 solar mass compact object, Astrophys. J. Lett. 896, L44 (2020).

[33] F. Fattoyev, C. Horowitz, J. Piekarewicz, and B. Reed, GW190814: Impact of a 2.6 solar mass neutron star on nucleonic equations of state, Phys. Rev. C 102, 065805 (2020).

[34] P. Danielewicz, R. Lacey, and W. G. Lynch, Determination of the equation of state of dense matter, Science 298, 1592 (2002).

[35] N.-B. Zhang and B.-A. Li, GW190814's secondary component with mass $2.50-2.67 \mathrm{M}_{\odot}$ as a superfast pulsar, Astrophys. J. 902, 38 (2020)

[36] A. Tsokaros, M. Ruiz, and S. L. Shapiro, GW190814: Spin and equation of state of a neutron star companion, Astrophys. J. 905, 48 (2020).

[37] I. Tews, P. T. H. Pang, T. Dietrich, M. W. Coughlin, S. Antier, M. Bulla, J. Heinzel, and L. Issa, On the nature of GW190814 and its impact on the understanding of supranuclear matter, Astrophys. J. Lett. 908, L1 (2021).

[38] V. Dexheimer, R. O. Gomes, T. Klähn, S. Han, and M. Salinas, GW190814 as a massive rapidly rotating neutron star with exotic degrees of freedom, Phys. Rev. C 103, 025808 (2021).

[39] K. Huang, J. Hu, Y. Zhang, and H. Shen, The possibility of the secondary object in GW190814 as a neutron star, Astrophys. J. 904, 39 (2020).

[40] A. Sedrakian, F. Weber, and J. J. Li, Confronting GW190814 with hyperonization in dense matter and hypernuclear compact stars, Phys. Rev. D 102, 041301(R) (2020).

[41] Z. Cao, L.-W. Chen, P.-C. Chu, and Y. Zhou, GW190814: Circumstantial evidence for up-down quark star, arXiv:2009.00942 (2020).

[42] C. Zhang and R. B. Mann, Unified interacting quark matter and its astrophysical implications, Phys. Rev. D 103, 063018 (2021).

[43] H. C. Das, A. Kumar, B. Kumar, S. K. Biswal, and S. K. Patra, The BigApple force and it's implications to finite nuclei and astrophysical objects, arXiv:2009.10690 (2020).

[44] T. Demircik, C. Ecker, and M. Järvinen, Rapidly spinning compact stars with deconfinement phase transition, Astrophys. J. Lett. 907, L37 (2021).

[45] V. B. Thapa, M. Sinha, J. J. Li, and A. Sedrakian, Equation of state of strongly magnetized matter with hyperons and $\Delta$ resonances, Particles 3, 660 (2020).

[46] I. Bombaci, A. Drago, D. Logoteta, G. Pagliara, and I. Vidaña, Was GW190814 a Black Hole-Strange Quark Star System? Phys. Rev. Lett. 126, 162702 (2021).

[47] B. Biswas, R. Nandi, P. Char, S. Bose, and N. Stergioulas, GW190814: On the properties of the secondary component of the binary, Mon. Not. Roy. Astron. Soc. 505, 1600 (2021).
[48] J. J. Li, A. Sedrakian, and F. Weber, Rapidly rotating $\Delta$ resonance-admixed hypernuclear compact stars, Phys. Lett. B 810, 135812 (2020).

[49] Z. Roupas, G. Panotopoulos, and I. Lopes, QCD color superconductivity in compact stars: Color-flavor locked quark star candidate for the gravitational-wave signal GW190814, Phys. Rev. D 103, 083015 (2021).

[50] V. B. Thapa and M. Sinha, Dense matter equation of state of a massive neutron star with antikaon condensation, Phys. Rev. D 102, 123007 (2020).

[51] I. A. Rather, A. A. Usmani, and S. K. Patra, Hadron-quark phase transition in the context of GW190814, J. Phys. G 48, 085201 (2021).

[52] A. Kanakis-Pegios, P. S. Koliogiannis, and C. C. Moustakidis, Probing the nuclear equation of state from the existence of a $\sim 2.6 M_{\odot}$ neutron star: The GW190814 puzzle, Symmetry 13, 183 (2021).

[53] D. Blaschke and M. Cierniak, Studying the onset of deconfinement with multi-messenger astronomy of neutron stars, Astron. Nachr. 342, 227 (2021).

[54] I. A. Rather, U. Rahaman, M. Imran, H. C. Das, A. A. Usmani, and S. K. Patra, Rotating Neutron stars with Quark cores, Phys. Rev. C 103, 055814 (2021).

[55] A. Ayriyan, D. Blaschke, A. G. Grunfeld, D. AlvarezCastillo, H. Grigorian, and V. Abgaryan, Bayesian analysis of multimessenger M-R data with interpolated hybrid EoS, arXiv:2102.13485 (2021).

[56] I. A. Rather, U. Rahaman, V. Dexheimer, A. A. Usmani, and S. K. Patra, Heavy magnetic neutron stars, Astrophys. J. 917, 46 (2021).

[57] C. Drischler, S. Han, J. M. Lattimer, M. Prakash, S. Reddy, and T. Zhao, Limiting masses and radii of neutron stars and their implications, Phys. Rev. C 103, 045808 (2021).

[58] J. L. Friar, D. Huber, and U. van Kolck, Chiral symmetry and three nucleon forces, Phys. Rev. C 59, 53 (1999).

[59] E. Epelbaum, Four-nucleon force in chiral effective field theory, Phys. Lett. B 639, 456 (2006).

[60] R. B. Wiringa, V. G. J. Stoks, and R. Schiavilla, An Accurate nucleon-nucleon potential with charge independence breaking, Phys. Rev. C 51, 38 (1995).

[61] M. Piarulli, I. Bombaci, D. Logoteta, A. Lovato, and R. B. Wiringa, Benchmark calculations of pure neutron matter with realistic nucleon-nucleon interactions, Phys. Rev. C 101, 045801 (2020).

[62] T. Lee and C.-N. Yang, Conservation of heavy particles and generalized gauge transformations, Phys. Rev. 98, 1501 (1955).

[63] L. Okun, Mirror particles and mirror matter: 50 years of speculations and search, Phys. Usp. 50, 380 (2007).

[64] A. E. Nelson and N. Tetradis, Constraints on a new vector boson coupled to baryons, Phys. Lett. B 221, 80 (1989).

[65] B. A. Dobrescu and C. Frugiuele, Hidden GeV-Scale Interactions of Quarks, Phys. Rev. Lett. 113, 061801 (2014).

[66] S. Gardner, R. J. Holt, and A. S. Tadepalli, New prospects in fixed target searches for dark forces with the SeaQuest experiment at Fermilab, Phys. Rev. D 93, 115015 (2016).

[67] D. C. Bailey and S. Davidson, Is there a vector boson coupling to baryon number? Phys. Lett. B 348, 185 (1995).

[68] C. D. Carone and H. Murayama, Possible Light U(1) Gauge Boson Coupled to Baryon Number, Phys. Rev. Lett. 74, 3122 (1995). 
[69] P. Fileviez Pérez and M. B. Wise, Baryon and lepton number as local gauge symmetries, Phys. Rev. D 82, 011901(R) (2010).

[70] S. Tulin, New weakly-coupled forces hidden in low-energy QCD, Phys. Rev. D 89, 114008 (2014).

[71] C. D. Carone and H. Murayama, Realistic models with a light U(1) gauge boson coupled to baryon number, Phys. Rev. D 52 , 484 (1995).

[72] J. D. Bjorken, R. Essig, P. Schuster, and N. Toro, New fixedtarget experiments to search for dark gauge forces, Phys. Rev. D 80, 075018 (2009).

[73] L. Michaels and F. Yu, Probing new $U(1)$ gauge symmetries via exotic $Z \rightarrow Z^{\prime} \gamma$ decays, J. High Energy Phys. 03 (2021) 120.

[74] R. Aaij et al. (LHCb collaboration), Search for dark photons produced in $13 \mathrm{TeV} p p$ collisions, Phys. Rev. Lett. 120, 061801 (2018).

[75] R. Foot, G. C. Joshi, and H. Lew, Gauged baryon and lepton numbers, Phys. Rev. D 40, 2487 (1989).

[76] X.-G. He and S. Rajpoot, Anomaly-free left-right-symmetric models with gauged baryon and lepton numbers, Phys. Rev. D 41, 1636 (1990).

[77] J. L. Feng, B. Fornal, I. Galon, S. Gardner, J. Smolinsky, T. M. P. Tait, and P. Tanedo, Particle physics models for the $17 \mathrm{MeV}$ anomaly in beryllium nuclear decays, Phys. Rev. D 95, 035017 (2017).

[78] X. G. He, G. C. Joshi, H. Lew, and R. R. Volkas, New-Z' phenomenology, Phys. Rev. D 43, R22(R) (1991).

[79] M. I. Haftel, Two-nucleon potentials and nuclear saturation, Ph.D. thesis, University of Pittsburgh, 1969.

[80] M. I. Haftel and F. Tabakin, Nuclear saturation and the smoothness of nucleon-nucleon potentials, Nucl. Phys. A 158 1 (1970).

[81] M. Hjorth-Jensen, T. Kuo, and E. Osnes, Realistic effective interactions for nuclear systems, Phys. Rep. 261, 125 (1995).

[82] Code available for download, https://www.phy.anl.gov/theory/ research/av18/.

[83] A. Akmal, V. R. Pandharipande, and D. G. Ravenhall, The Equation of state of nucleon matter and neutron star structure, Phys. Rev. C 58, 1804 (1998).

[84] For reference, we note that the study of Ref. [61] shows the many-body method variation is far smaller for the simpler AV6' force.

[85] L. McLerran and S. Reddy, Quarkyonic Matter and Neutron Stars, Phys. Rev. Lett. 122, 122701 (2019).

[86] P. Landry, R. Essick, and K. Chatziioannou, Nonparametric constraints on neutron star matter with existing and upcoming gravitational wave and pulsar observations, Phys. Rev. D 101, 123007 (2020).

[87] The uncertainties on and correlations between the parameters of the AV18 potential have not been provided. Therefore, we are unable to quantify the uncertainties on any predicted quantities.

[88] P. Demorest, T. Pennucci, S. Ransom, M. Roberts, and J. Hessels, Shapiro delay measurement of a two solar mass neutron star, Nature (London) 467, 1081 (2010).

[89] J. Antoniadis et al., A Massive Pulsar in a Compact Relativistic Binary, Science 340, 1233232 (2013).

[90] Z. Arzoumanian, A. Brazier, S. Burke-Spolaor, S. Chamberlin, S. Chatterjee, B. Christy, J. M. Cordes, N. J. Cornish, F. Crawford, H. T. Cromartie et al. (NANOGrav), The
NANOGrav 11-year Data Set: High-precision timing of 45 Millisecond Pulsars, Astrophys. J. Suppl. 235, 37 (2018).

[91] H. T. Cromartie, E. Fonseca, S. M. Ransom, P. B. Demorest, Z. Arzoumanian, H. Blumer, P. R. Brook, M. E. DeCesar, T. Dolch, J. A. Ellis et al. (NANOGrav), Relativistic Shapiro delay measurements of an extremely massive millisecond pulsar, Nature Astron. 4, 72 (2019).

[92] We have considered the high-density phase EoS presented in this work, which includes the effects of neutral pion condensation. This phase dominates the structure of NSs with $M \gtrsim 0.5 M_{\odot}$; we employ it universally in our analysis.

[93] S. Koranda, N. Stergioulas, and J. L. Friedman, Upper limit set by causality on the rotation and mass of uniformly rotating relativistic stars, Astrophys. J. 488, 799 (1997).

[94] H. Tan, J. Noronha-Hostler, and N. Yunes, Neutron Star Equation of State in light of GW190814, Phys. Rev. Lett. 125, 261104 (2020).

[95] G. A. Lalazissis, J. Konig, and P. Ring, A New parametrization for the Lagrangian density of relativistic mean field theory, Phys. Rev. C 55, 540 (1997).

[96] F. J. Fattoyev, C. J. Horowitz, J. Piekarewicz, and G. Shen, Relativistic effective interaction for nuclei, giant resonances, and neutron stars, Phys. Rev. C 82, 055803 (2010).

[97] W.-C. Chen and J. Piekarewicz, Searching for isovector signatures in the neutron-rich oxygen and calcium isotopes, Phys. Lett. B 748, 284 (2015).

[98] L. Ametller, J. Bijnens, A. Bramon, and F. Cornet, Chiral perturbation theory for $\eta \rightarrow \pi^{0} \gamma \gamma$, Phys. Lett. B 276, 185 (1992).

[99] J. Bijnens, A. Fayyazuddin, and J. Prades, The $\gamma \gamma \rightarrow \pi^{0} \pi^{0}$ and $\eta \rightarrow \pi^{0} \gamma \gamma$ transitions in the extended NJL model, Phys. Lett. B 379, 209 (1996).

[100] E. Oset, J. R. Pelaez, and L. Roca, $\eta \rightarrow \pi^{0} \gamma \gamma$ decay within a chiral unitary approach, Phys. Rev. D 67, 073013 (2003).

[101] E. Oset, J. R. Peláez, and L. Roca, $\eta \rightarrow \pi^{0} \gamma \gamma$ decay within a chiral unitary approach revisited, Phys. Rev. D 77, 073001 (2008).

[102] R. Escribano, S. Gonzàlez-Solís, R. Jora, and E. Royo, Theoretical analysis of the doubly radiative decays $\eta^{(\prime)} \rightarrow \pi^{0} \gamma \gamma$ and $\eta^{\prime} \rightarrow \eta \gamma \gamma$, Phys. Rev. D 102, 034026 (2020).

[103] P. A. Zyla, R. M. Barnett, J. Beringer, O. Dahl, D. A. Dwyer, D. E. Groom, C.-J. Lin, K. S. Lugovsky, E. Pianori, D. J. Robinson et al. (Particle Data Group), Review of particle physics, Prog. Theor. Exp. Phys. 2020, 083 C01 (2020).

[104] R. Barbieri and T. E. O. Ericson, Evidence against the existence of a low mass scalar boson from neutron-nucleus scattering, Phys. Lett. B 57, 270 (1975).

[105] H. Leeb and J. Schmiedmayer, Constraint on Hypothetical Light Interacting Bosons From Low-Energy Neutron Experiments, Phys. Rev. Lett. 68, 1472 (1992).

[106] M. Bando, T. Kugo, S. Uehara, K. Yamawaki, and T. Yanagida, Is rho Meson a Dynamical Gauge Boson of Hidden Local Symmetry? Phys. Rev. Lett. 54, 1215 (1985).

[107] M. Bando, T. Kugo, and K. Yamawaki, On the vector mesons as dynamical gauge bosons of hidden local symmetries, Nucl. Phys. B 259, 493 (1985).

[108] T. Fujiwara, T. Kugo, H. Terao, S. Uehara, and K. Yamawaki, Non-abelian anomaly and vector mesons as dynamical gauge bosons of hidden local symmetries, Prog. Theor. Phys. 73, 926 (1985). 
[109] M. Bando, T. Kugo, and K. Yamawaki, Nonlinear realization and hidden local symmetries, Phys. Rep. 164, 217 (1988).

[110] L. Gan et al., Symmetry Tests of Rare Eta Decays to All-Neutral Final States: The JLab Eta Factory (JEF) Experiment, available at https://www.jlab.org/exp_prog/proposals/ 14/PR12-14-004.pdf.

[111] L. Gan et al., Update to the JEF proposal (PR12-14-004), available at https://misportal.jlab.org/pacProposals/proposals/ 1354/attachments/98421/Proposal.pdf.

[112] L. Gan, B. Kubis, E. Passemar, and S. Tulin, Precision tests of fundamental physics with $\eta$ and $\eta^{\prime}$ mesons, arXiv:2007.00664 (2020).

[113] M. Ablikim, M. N. Achasov, S. Ahmed, X. C. Ai, O. Albayrak, M. Albrecht, D. J. Ambrose, A. Amoroso, F. F. An, Q. An et al. (BESIII Collaboration), Observation of the doubly radiative decay $\eta^{\prime} \rightarrow \gamma \gamma \pi^{0}$, Phys. Rev. D 96, 012005 (2017).

[114] M. Ablikim, M. N. Achasov, S. Ahmed, M. Albrecht, M. Alekseev, A. Amoroso, F. F. An, Q. An, Y. Bai, O. Bakina et al. (BESIII Collaboration), Precision Measurement of the Branching Fractions of $\eta$ ' Decays, Phys. Rev. Lett. 122, 142002 (2019).

[115] M. Ablikim, M. N. Achasov, P. Adlarson, S. Ahmed, M. Albrecht, M. Alekseev, A. Amoroso, F. F. An, Q. An, Y. Bai et al. (BESIII Collaboration), Search for the decay $\eta^{\prime} \rightarrow \gamma \gamma \eta$, Phys. Rev. D 100, 052015 (2019).

[116] J. L. Rosner, Quark content of neutral mesons, Phys. Rev. D 27, 1101 (1983).

[117] E. Kou, On the $\eta^{\prime}$ gluonic admixture, Phys. Rev. D 63, 054027 (2001).

[118] R. Escribano and J. Nadal, On the gluon content of the $\eta$ and $\eta^{\prime}$ mesons, J. High Energy Phys. 05 (2007) 006.

[119] F. Ambrosinod, A. Antonellia, M. Antonellia, C. Baccii, P. Beltrameb, G. Bencivennia, S. Bertoluccia, C. Binig, C. Bloisea, S. Bocchettai et al. (KLOE Collaboration), Measurement of the pseudoscalar mixing angle and $\eta^{\prime}$ gluonium content with the KLOE detector, Phys. Lett. B 648, 267 (2007).

[120] C. Gatto, B. Fabela Enriquez, and M. I. Pedraza Morales (REDTOP), The REDTOP project: Rare eta decays with a TPC for optical photons, PoS (ICHEP2016), 812 (2016).

[121] D. González, D. León, B. Fabela, and M. I. Pedraza, Detecting physics beyond the Standard Model with the REDTOP experiment, J. Phys.: Conf. Ser. 912, 012042 (2017).
[122] C. Gatto (REDTOP), The REDTOP experiment, arXiv:1910.08505 (2019).

[123] S. Adhikari, C. S. Akondi, H. Al Ghoul, A. Ali, M. Amaryan, E. G. Anassontzis, A. Austregesilo, F. Barbosa, J. Barlow, A. Barnes et al. (GlueX), The GLUEX beamline and detector, Nucl. Instrum. Meth. A 987, 164807 (2021).

[124] C. Fanelli and M. Williams, Photoproduction of leptophobic bosons, J. Phys. G 44, 014002 (2017).

[125] M. I. Krivoruchenko, F. Šimkovic, and A. Faessler, Constraints for weakly interacting light bosons from existence of massive neutron stars, Phys. Rev. D 79, 125023 (2009).

[126] D. Gerstung, N. Kaiser, and W. Weise, Hyperon-nucleon three-body forces and strangeness in neutron stars, Eur. Phys. J. A 56, 175 (2020).

[127] G. B. Cook, S. L. Shapiro, and S. A. Teukolsky, Rapidly rotating neutron stars in general relativity: Realistic equations of state, Astrophys. J. 424, 823 (1994).

[128] J.-P. Lasota, P. Haensel, and M. A. Abramowicz, Fast rotation of neutron stars, Astrophys. J. 456, 300 (1996).

[129] C. Breu and L. Rezzolla, Maximum mass, moment of inertia and compactness of relativistic stars, Mon. Not. Roy. Astron. Soc. 459, 646 (2016).

[130] P. S. Koliogiannis and C. C. Moustakidis, Effects of the equation of state on the bulk properties of maximally-rotating neutron stars, Phys. Rev. C 101, 015805 (2020).

[131] E. R. Most, L. J. Papenfort, L. R. Weih, and L. Rezzolla, A lower bound on the maximum mass if the secondary in GW190814 was once a rapidly spinning neutron star, Mon. Not. Roy. Astron. Soc. 499, L82 (2020).

[132] R. Essick and P. Landry, Discriminating between neutron stars and black holes with imperfect knowledge of the maximum neutron star mass, Astrophys. J. 904, 80 (2020).

[133] T. W. Baumgarte, S. L. Shapiro, and M. Shibata, On the maximum mass of differentially rotating neutron stars, Astrophys. J. Lett. 528, L29 (2000).

[134] D. Gondek-Rosińska, I. Kowalska, L. Villain, M. Ansorg, and M. Kucaba, A new view on the maximum mass of differentially rotating neutron stars, Astrophys. J. 837, 58 (2017).

[135] L. R. Weih, E. R. Most, and L. Rezzolla, On the stability and maximum mass of differentially rotating relativistic stars, Mon. Not. Roy. Astron. Soc. 473, L126 (2018). 